\title{
Individual Differences in Attentional Bias Associated with Cocaine Dependence Are Related to Varying Engagement of Neural Processing Networks
}

\author{
Clint D Kilts*,', Ashley Kennedy', Amanda L Elton', Shanti Prakash Tripathi', Jonathan Young', \\ Josh M Cisler' and G Andrew James' \\ 'Brain Imaging Research Center, Psychiatric Research Institute, College of Medicine, University of Arkansas for Medical Sciences, \\ Little Rock, AR, USA
}

\begin{abstract}
Cocaine and other drug dependencies are associated with significant attentional bias for drug use stimuli that represents a candidate cognitive marker of drug dependence and treatment outcomes. We explored, using $\mathrm{MMRI}$, the role of discrete neural processing networks in the representation of individual differences in the drug attentional bias effect associated with cocaine dependence (AB-coc) using a word counting Stroop task with personalized cocaine use stimuli (cocStroop). The cocStroop behavioral and neural responses were further compared with those associated with a negative emotional word Stroop task (eStroop) and a neutral word counting Stroop task (cStroop). Brain-behavior correlations were explored using both network-level correlation analysis following independent component analysis (ICA) and voxel-level, brain-wide univariate correlation analysis. Variation in the attentional bias effect for cocaine use stimuli among cocaine-dependent men and women was related to the recruitment of two separate neural processing networks related to stimulus attention and salience attribution (inferior frontal-parietal-ventral insula), and the processing of the negative affective properties of cocaine stimuli (frontal-temporal-cingulate). Recruitment of a sensory-motor-dorsal insula network was negatively correlated with $\mathrm{AB}-\mathrm{coc}$ and suggested a regulatory role related to the sensorimotor processing of cocaine stimuli. The attentional bias effect for cocaine stimuli and for negative affective word stimuli were significantly correlated across individuals, and both were correlated with the activity of the frontal-temporal-cingulate network. Functional connectivity for a single prefrontal-striatal-occipital network correlated with variation in general cognitive control (cStroop) that was unrelated to behavioral or neural network correlates of cocStroop- or eStroop-related attentional bias. A brain-wide mass univariate analysis demonstrated the significant correlation of individual attentional bias effect for cocaine stimuli with distributed activations in the frontal, occipitotemporal, parietal, cingulate, and premotor cortex. These findings support the involvement of multiple processes and brain networks in mediating individual differences in risk for relapse associated with drug dependence.
\end{abstract}

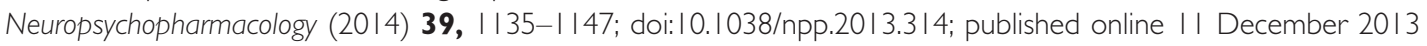

Keywords: cocaine; attentional bias; functional magnetic resonance imaging; individual differences; neural networks; independent component analysis

\section{INTRODUCTION}

A pathological motivation for drug use in spite of adverse consequences is a cardinal feature of drug dependence. The conditioned association of drug use with people, places, and things renders these drug-related stimuli as having conditioned incentive motivation. Exposure to such drug stimuli results in drug craving states and thus represents a major mechanism of relapse to drug seeking and use behaviors in drug-dependent individuals (Paliwal et al, 2008; Shadel

\footnotetext{
*Correspondence: Dr CD Kilts, Psychiatric Research Institute, University of Arkansas for Medical Sciences, 430I West Markham Street, Mail Slot 554, Little Rock, AR 72205, USA, Tel: + I 501 5268163, Fax: + I 5015268199, E-mail: cdkilts@uams.edu Received 3 July 2013; revised 3 October 2013; accepted 23 October 2013; accepted article preview online 7 November 2013
}

et al, 2011). The conditioned incentive motivation for drug use stimuli in drug-dependent individuals has been demonstrated using implicit stimulus processing tasks such as drug Stroop (Cox et al, 2006) and dot probe (Bradley et al, 2003) task paradigms as well as stimulus viewing preference (Moeller et al, 2009). These performance-based tasks are referred to as measures of attentional bias for drug use-related stimuli. Drug dependence-related attentional bias is thought to represent a maladaptive form of valuedriven attentional capture (Anderson et al, 2011) in which stimuli imbued with value via associative learning capture attention. Recent research demonstrates that these conditioned motivational responses are associated with momentary temptations to use drugs (Waters et al, 2012), and predict more temporally extended drug craving, treatment adherence, and relapse across diverse drugs of abuse, 
including cocaine (Carpenter et al, 2006; Copersino et al, 2004; Hester et al, 2006; Vadhan et al, 2007).

Based on these outcomes, attentional bias for drug-related stimuli has been proposed to represent a cognitive marker of drug addiction, although it remains unclear as to whether attentional bias represents a trait or state marker (Ataya et al, 2012). Moreover, although little discussed, the magnitude of attentional bias exhibits marked individual variability with drug stimuli responses varying between large positive attentional bias to negative attentional bias in which the reaction time to drug-neutral trials exceeds that of drug stimulus trials (Carpenter et al, 2012; Janes et al, 2010). This variability in incentive salience attribution to drug stimuli suggests wide individual variation in risk for relapse among drug-dependent individuals. Recent functional neuroimaging studies have sought to characterize the neural processing events that underlie attentional bias related to drug dependence as a means of understanding the mechanisms of relapse. Such study designs are distinct from those exploring the neural correlates of drug cue reactivity in the absence of assessing their cognitive bias properties by task interference effects (Kilts et al, 2001; Potenza et al, 2012). For nicotine-dependent individuals, the level of attentional bias for smoking-related cues was positively correlated with activation of the limbic, paralimbic, and occipital brain regions associated with emotional salience, memory recall, interoceptive states, and visual processing (Janes et al, 2010). Stimulant-dependent individuals exhibited a significant attentional bias for drug-related word stimuli that was significantly correlated with greater activation of the left ventral prefrontal cortex and cerebellum (Ersche et al, 2010). Moreover, the right dorsal anterior cingulate cortex response to cocaine use-related stimuli predicted recent cocaine use at 3-month follow-up in treatment-engaged cocaine-dependent individuals (Marhe et al, 2013). These findings support the contention that attentional bias and its associated neural correlates represent neurocognitive markers of drug dependence and individual risk for relapse. We sought to further characterize the neural information processing related to individual variation in attentional bias in cocaine-dependent adults at the level of the involved neural processing networks as this brain state representation has increased power to describe individual differences in brain-behavior relationships relative to more traditional univariate, voxel-wise analyses (Congdon et al, 2010; Pantazatos et al, 2012).

In the present study, we sought to test a hypothesis that individual differences in $A B-\operatorname{coc}$ are related to the relative activity across spatially independent networks of activation and distinct from network activity related to attentional bias for emotionally negative words (AB-neg). To support hypothesis testing, an independent component analysis (ICA) was used to identify spatially independent networks of activated brain regions to accomplish a goal of reducing the dimensionality of the highly complex feature space of fMRI data sets (Calhoun et al, 2001; Beckmann and Smith, 2004). Regression analysis further identified the relationship between component (network) activity and individual variation in attentional bias for personalized cocaine use reminders. This relationship was also assessed controlling for variables of no interest (eg, age, ethnicity, and so on). We also similarly assessed the relationship of individual differences in cocaine attentional bias with neural processing networks related to general (nondrug) cognitive control as neural responses to a classical color-word Stroop task predicted treatment completion among cocaine-dependent individuals (Brewer et al, 2008). Further comparisons were made related to network activity and individual variation in attentional bias for emotionally negative words to characterize dysphoric states as another precipitant of relapse in drug-dependent individuals (Koob, 2009). The results obtained support a view that individual variation in attentional bias task estimates of drug incentive motivation are related to the variation in the coherence of activation in discrete large-scale neural processing networks. The ICA-based analysis further suggested that network activity corresponds to regulatory, affective, and motivational variation in responses to cocaine cues.

\section{MATERIALS AND METHODS}

\section{Participants}

A sample of 42 men and women (26\%) who fulfilled DSMIV syndromal criteria for cocaine dependence defined by structured clinical interview (SCID; First et al, 1993) provided written informed consent to participate in a clinical trial approved by the UAMS Institutional Review Board. The presence of current mood, anxiety, or psychotic disorders were exclusionary for study participation. The study sample was treatment-seeking with clinical and MRI assessments collected before randomization to study treatment arms. Demographic and clinical characteristics of the study participants are described in Table 1.

\section{Tasks}

During fMRI, participants completed three variants of a word counting Stroop task (Bush et al, 1998): a counting Stroop task (cStroop) using drug-unrelated words to assess global cognitive control ability, a drug Stroop task (Cox et al, 2006) involving cocaine use-related words (cocStroop) to assess cocaine attentional bias, and an emotional Stroop task (eStroop) involving negative emotional words to assess the role of negative affect in the cocaine attentional bias effect. The drug use stimuli corresponded to six words provided by the participants that were associated with their drug use, and were thus personalized. Participants rankordered the words based on their perceived significance as drug use reminders.

Tasks were conducted across three fMRI runs within a single session, with each run including trials from the three Stroop task variants. Each trial presented 1-4 identical words in a vertical array, with participants instructed to indicate the number of words presented by pressing one of four preassigned buttons on a MRI-compatible response device (Current Designs) as quickly as possible. Stimuli were presented for $2000 \mathrm{~ms}$ followed by the presentation of a fixation cross with an interstimulus interval of $300 \mathrm{~ms}$. Task training before fMRI acquisition involved repeated trials of the cStroop task until an accuracy of $\geqslant 95 \%$ was attained. Word stimulus trials representing the cStroop, eStroop, and cocStroop tasks were presented in a mixed blocked, event-related design in a pseudorandom order with 
Table I Descriptive Clinical, Demographic, and Functional Characteristics and Task Performance for the Sample of Cocaine-Dependent Participants

$\begin{array}{lc}\text { Demographic variables } & \\ N & 42 \\ \text { Age } & 44.0 \pm 8.7 \\ \text { Sex (F/M) } & 1 / / 3 \mid \\ \text { Race (AA/CA/H) } & 33 / 8 / 1 \\ \text { Education (years) } & 12.4 \pm 2.0 \\ \text { Handedness (R/L) } & 39 / 3 \\ & \\ \text { Cocaine use } & 39 / 3 \\ \text { Route of cocaine use (smoke/snort) } & 27.9 \pm 6.9 \\ \text { Age of daily cocaine use } & 2.8 \pm 2.3 \\ \text { Cocaine use: days in past } 7 \text { days } & 13.5 \pm 9.4 \\ \text { Cocaine use: days in past } 30 \text { days }\end{array}$

Clinical variables (no. current/past)

Major depression

Alcohol dependence

Alcohol abuse

Marijuana dependence

Marijuana abuse

Nicotine use: daily $/>||$ years

\section{Addiction severity index (ASI)}

Medical

$0.19 \pm 0.26$

Employment

$0.69 \pm 0.27$

Drug

$0.20 \pm 0.10$

Alcohol

$0.22 \pm 0.27$

Legal

$0.10 \pm 0.15$

Psychiatric

$0.05 \pm 0.20$

Family/social

$0.15 \pm 0.20$

$\begin{array}{ll}\text { Quantitative assessments (total scores) } & \\ \text { BIS-II } & 68.2 \pm 11.0 \\ \text { CTQ } & 45.2 \pm 17.9\end{array}$

Task performance (RT/accuracy)

Cocaine

$1028 \pm 154 / 92.1 \%$

Emotional

$980 \pm 148 / 93.0 \%$

Neutral

$955 \pm|5| / 92.8 \%$

Incongruent

$1015 \pm 164 / 90.1 \%$

Congruent

$855 \pm 147 / 95.3 \%$

Abbreviations: AA, African-American; BIS-II, Barratt Impulsiveness Scale; CA Caucasian; CTQ, Childhood Trauma Questionnaire; H, Hispanic; RT, reaction time (ms).

a total run time of $\sim 12 \mathrm{~min}$. Each run consisted of 3 blocks of word counting trials with each block separated by $20 \mathrm{~s}$ of fixation cross-presentation. The first and third blocks of stimulus trials each consisted of 16 eStroop trials, 16 cocStroop trials, and 66 neutral word trials. The second block consisted of 62 congruent cStroop trials (where the number of words matched the word shown, such as 'three' printed 3 times) and 34 incongruent cStroop trials (where the number of words did not match the word displayed, such as 'two' presented 4 times). The fMRI session involved three runs with a 1-min rest period between each run for a total on-task scanner time of $\sim 38 \mathrm{~min}$. Stroop task-related fMRI responses and behavioral measures of reaction time (RT) and response accuracy were collected for each task trial during the fMRI session.

\section{Drug Use Cue Word Generation}

The Cocaine Craving Cue Words Generator was used to obtain six or more personalized cue words that 'trigger' a craving to use cocaine in the individual being assessed. The words were generated in an interview format in which the participants were asked to think of any triggers such as familiar sights, sounds, smells, or feelings that reminded them of situations where they used crack cocaine. Once an image was formed in the mind of the participants, the interviewer would ask them to try to list at least 6 and up to 12 cocaine-trigger words from those images. Participants listed an average of 9 words each. If the participants could not come up with at least 6 words, the interviewer would guide them toward certain trigger words that were still personal to the individual. These words typically included objects used to smoke crack cocaine, first names or nicknames of their dealers or friends they used cocaine with, places where they frequently used cocaine, or sensations associated with using cocaine. After the participants listed 6-12 cocaine trigger words, they were asked to choose the 6 words that were most closely related to their craving of crack cocaine. As participants chose these words, they were asked to think about how each individual word affected their craving for crack cocaine and then rate them with 1 being the strongest trigger word and 6 being the weakest. Similarly, the negative emotional word list consisted of eight words with negative affect connotation (eg, painful, hazard) that were taken from a word list used in prior studies of implicit emotion processing (Whalen et al, 1998); with the exclusion of emotional words (eg, jail) that are often associated with cocaine use. The neutral word list consisted of eight words (eg, desk, phone) assessed as unrelated to the individual's cocaine use or negative affect word list. The different word stimuli were not matched for lexical properties such as length, part of speech, or frequency of use in the English language.

\section{fMRI Acquisition}

fMRI data were acquired $6 \pm 7$ (range 1-29) days following the generation of cocaine cue words at the Brain Imaging Research Center (BIRC). Study participants completed the word counting tasks while undergoing scanning in a Phillips 3T Achieva X-series MRI scanner with an 8-channel head coil. fMRI data were collected using an echo planar (EPI) sequence with thin axial slices ( $3 \mathrm{~mm}$ thickness) to minimize signal loss in susceptible regions of interest (eg, ventral prefrontal cortex). The EPI sequence used the following parameters: $\mathrm{TR} / \mathrm{TE} / \mathrm{FA}=2000 \mathrm{~ms} / 30 \mathrm{~ms} / 90^{\circ}, \quad$ matrix $=64$ $\times 64$ with $\mathrm{FOV}=192 \times 192 \mathrm{~mm}$ for final resolution $3 \times$ $3 \times 3 \mathrm{~mm}, 30$ axial slices. Structural scout images were acquired using a spin-echo, T1-weighted pulse sequence 
$\left(\mathrm{TR} / \mathrm{TE} / \mathrm{FA}=500 \mathrm{~ms} / 20 \mathrm{~ms} / 90^{\circ}\right)$. A magnetization prepared gradient echo sequence (MPRAGE) was used to acquire 3D anatomic data to enable activation localization and coregistration with functional neuroimaging data (matrix $=$ $256 \times 256,160$ sagittal slices, $T R / T E / F A=2600 \mathrm{~ms} / 3.02 \mathrm{~ms} / 8^{\circ}$, final resolution $=1 \times 1 \times 1 \mathrm{~mm}^{3}$ resolution).

\section{fMRI Preprocessing}

Image processing and analysis was conducted with SAS, AFNI (Cox, 1996), and Matlab (The MathWorks). Functional images underwent slice timing correction, deobliquing, motion-correction by registration to the tenth image acquired for each run using a 6-parameter transformation (Friston et al, 1995), spatial normalization to the Montreal Neurological Institute (MNI) template via 12-parameter affine transformation followed by nonlinear warping using basis functions (Ashburner and Friston, 1999), regression of mean signal from cerebrospinal fluid (CSF) and white matter, spatial smoothing with a Gaussian kernel $(5 \mathrm{~mm}$ FWHM) to enhance signal-to-noise ratios and enable group comparisons, quadratic detrending, and scaling to percent signal change.

fMRI artifacts because of head motion were pronounced among this sample and increasingly prominent throughout the session. The final preprocessing step used ICA to model and remove noise due to head movement. Gross head motion could lead to a shift in the intensity encoding of brain slices (based upon slice acquisition) that in turn would manifest in a seed connectivity map as alternating 'stripes' of signal intensity. To correct for this artifact, an ICA was performed for each fMRI data set using the Group ICA Functional Toolbox (GIFT v1.3) and the following GIFT options: principal component analysis (PCA) solving for 224 PCA components using expectation maximization, no further reduction into ICA components, back-reconstruction using GICA3, and estimation of components with intensity normalization. (Please see 'Multivariate fMRI data' below for further details about GIFT and ICA.) Because head motion leads to a shift in slice encoding intensity, components related to head motion manifest spatially as stripes contributing temporal variance to the overall signal. As striping patterns of brain activity are not biologically plausible, a strict threshold was selected to identify components that correlated with slice acquisition patterns $(r \geq=0.07)$. These artifact components (if any) were identified and subtracted from the overall signal using Remove Components utility of GIFT.

After motion-related artifact removal, functional images for 9 of 51 participants (18\%) still had motion artifacts (striping) in their seed connectivity maps for run 1 . Of the 42 participants with usable data from the first fMRI run, only $16(38 \%)$ had usable data from all three runs. Consequently, only data from the first fMRI run were analyzed, as the larger sample size offered greater generalizability of results and supported the intended individual differences research.

\section{fMRI Data Analysis}

The brain-behavior relationship underlying individual differences in attentional bias for drug use reminders associated with cocaine dependence was explored with both ICAsupported neural processing network analysis and traditional univariate voxel-wise analysis. Multiple linear regression was used to assess the extent of variance in the attentional bias effect explained by variance in the activity of correlated neural processing networks controlling for variables of no interest (ie, age, sex, recent cocaine use, impulsiveness). Stepwise selection criteria were used to achieve the most parsimonious model that explained maximum variance.

Multivariate $f M R I$ data. After preprocessing, group-level ICA of functional image data sets were conducted using Matlab and the Group ICA of fMRI Toolbox (GIFT v1.3; http://icatb.sourceforge.net). ICA is an approach for blindsource separation of a complex mixture of signals and noise into spatially and temporally distinct components/signals. The number of components for which to solve was determined through permutation testing. We ran ICA solving for 20, 30, 40 , and 50 components and derived a stability index for each solution. The stability index was greatest for 30 components; solving for fewer components led to overlapping networks (ie, less compactness of clusters) and solving for more components led to fragmented components.

ICA was performed using publicly available Matlab scripts (http://mialab.mrn.org/software/) as follows. First, a two-step data-reduction approach was performed using PCA. The first stage reduced each subject's data set into 50 principal components (using expectation maximization and stacked data set options), and the second stage reduced these $42 \times 50=2100$ components into 30 group-level principal components. Next, the ICASSO toolbox was used to determine the reliability of these ICA components. ICASSO was repeated 30 times using the Infomax algorithm to calculate the stability index (iq) of each component across iterations. Then, back-reconstruction was performed using the GICA3 algorithm to identify each component's representation within each participant. Finally, all data were scaled to $z$-scores. Additional software parameters included full storage of covariance matrix to double precision, usage of selective eigenvariate solvers, and intensity normalization, as detailed in the GIFT v1.3 User Manual.

Multiple regression sorting criteria were used to explain the temporal sorting components in GIFT and derive fit regression parameters. Temporal sorting of components used the canonical hemodynamic response function (with amplitude modulation by trial RT) to model the expected neuronal response to each condition of interest (eg, cocaine $v s$ neutral word trials), and evaluated the fit ( $\beta$-weights) within-participant of each ICA-derived components' weighted activity time course to those conditions. This first step identified components whose activity ( $\beta$-weights) was significantly related to task ( $p<0.05$, uncorrected). Second, least squares linear regression (SAS 9.3) was performed for components surviving the first step of analysis to determine the relationship between components' $\beta$-weights and behavioral performance on task conditions (eg, cocaine $v s$ neutral word trials) across participants. This second step used false discovery rate (FDR) multiple comparison correction to control for the number of components surviving the first step. The group means for the averaged 
$\beta$-weights for each component for each task condition and planned contrast between conditions are defined in Table 2 .

Univariate analyses. Conventional univariate analyses were also conducted. GLM analyzed the voxel-wise relationship of brain activity to task condition for each participant as described above, followed by mixed-effects meta-analysis (MEMA) to determine group-level voxel activity. MEMA results were thresholded using 3dClustSim of AFNI: an uncorrected $p$-value of $<0.005$ and cluster size of $>18$ voxels were used to identify clusters significantly activated by task $\left(p_{\text {corr }}=0.05\right)$. Linear regression related their mean $\beta$-weights to behavioral task performance measures across participants.

\section{Behavioral Data Analysis}

Task performance data analyses were conducted with SAS. Mean participant reaction time was calculated for each condition (congruent cStroop, incongruent cStroop, cocStroop, eStroop, neutral). Interference effects were calculated for the cocStroop (mean cocStroop trial RT minus mean neutral trial RT), eStroop (mean eStroop trial RT minus mean neutral trial RT), and cStroop (mean incongruent trial RT minus mean congruent trial RT). Differences in interference effects between conditions were calculated with ANOVA (or its nonparametric version for variables lacking a Gaussian distribution), with post hoc tests determining significant $(p<0.05)$ differences between condition types. Spearman's correlation analyses assessed the significance of associations between individual attentional bias effect for cocaine words and recent cocaine use (past 7 or 30 days) and addiction-related disability (Addiction Severity Index (ASI) total score).

\section{RESULTS}

A total of 42 cocaine-dependent men $(n=31)$ and women $(n=11)$ participated in the study (Table 1$)$. The sample was middle aged ( $44 \pm 8.7$ years, mean $\pm \mathrm{SD})$ and racially diverse

Table 2 Component $\beta$ - Weights (Depicted as Group Mean (SD)) for Each Task Condition and Contrast $(N=42)$

\begin{tabular}{lcccc}
\hline & Comp 6 & Comp I2 & Comp I9 & Comp 2 I \\
\hline Cocaine- $\beta$ & $-0.17(1.65)$ & $-0.07(1.70)$ & $-0.16(2.12)$ & $1.80(1.82)$ \\
Neutral- $\beta$ & $0.47(1.71)$ & $-0.98(1.32)$ & $-0.17(1.56)$ & $0.48(1.60)$ \\
Emotional- $\beta$ & $0.68(1.67)$ & $-0.51(2.02)$ & $-0.40(1.94)$ & $0.45(2.02)$ \\
Incongruent- $\beta$ & $0.50(2.26)$ & $-0.46(2.27)$ & $-0.51(2.61)$ & $1.44(2.40)$ \\
Congruent $\beta$ & $0.65(1.96)$ & $-0.31(1.86)$ & $-0.63(2.25)$ & $0.97(2.30)$ \\
Coc- $\beta$ vs Neut- $\beta$ & $-0.64(1.33)$ & $0.91(1.51)$ & $0.011(1.41)$ & $1.32(1.45)$ \\
$t$-value & $-3.12 * *$ & $3.93 * * *$ & 0.05 & $5.83^{* * * *}$ \\
Emo- $\beta$ vs Neut $\beta$ & $0.21(1.14)$ & $0.48(1.44)$ & $-0.23(1.34)$ & $-0.03(1.21)$ \\
$t-$-value & 1.20 & $2.14 *$ & -1.12 & -0.16 \\
Incon- $\beta$ vs Con- $\beta$ & $-0.16(1.20)$ & $-0.16(1.10)$ & $0.12(0.93)$ & $0.47(0.96)$ \\
$t$-value & -0.86 & -0.93 & 0.82 & $3.13 * *$ \\
\hline
\end{tabular}

* $p<0.05, * * * 0.01$, **** $p$-value $<0.001$.
(33 African-American, 8 Caucasian, and 1 Hispanic). An additional 9 participants were recruited, but excessive head motion precluded the use of their neuroimaging data; their data are not included in these analyses. Subjects exhibited an onset of daily cocaine use in early adulthood and selfreported cocaine use in the past 7 and 30 days. The subjects were treatment seeking, although over $40 \%$ either failed to enroll $(n=12)$ or discontinued behavioral treatment early ( $<2$ sessions) $(n=6)$. The participants exhibited functional drug use-related impairment as assessed by the ASI. Moreover, as a group the participants had histories of childhood maltreatment (>90th percentile scores; Scher et al, 2001) and exhibited elevated levels of impulsiveness similar to previous studies of cocaine-dependent samples (Stanford et al, 2009). Of the 42 participants, 34 were positive for cocaine before fMRI whereas 8 were negative for cocaine by urinalysis. Of the 34 cocaine-positive subjects, 26 were positive for cocaine only, 7 were positive for cocaine and tetrahydocannabinol (THC), and 1 was positive for cocaine and benzodiazepines. One cocaine-negative subject was positive for THC. The subject sample also included 9 individuals who fulfilled DSM-IV criteria for current alcohol dependence, 7 with current alcohol abuse, 2 with current marijuana dependence, and 7 fulfilled criteria for current marijuana abuse. Two subjects fulfilled criteria for current opiate abuse. Although nicotine dependence was not directly assessed, from the ASI items 34 participants reported smoking for $21-30$ of the past 30 days; 32 reported smoking for the past $11+$ years. Only 5 participants reported never having smoked.

\section{Cocaine, Emotional, and Counting Stroop Task Performance}

A mean word counting accuracy of $90.1 \pm 10.1 \%$ (incongruent trials) to $95.3 \pm 11.8 \%$ (congruent trials) was observed across the five task conditions (Table 1); response accuracy did not significantly influence the brain-behavior relationships (all $p$ 's $>0.05$ ). Box plots (Figure 1) of reaction time difference for cocaine or emotional word stimuli relative to neutral words, and for incongruent $v s$ congruent word stimuli, illustrate a significant group main effect of word type $(\mathrm{F}=32.63, \mathrm{df}=2, p<0.0001)$. For all types, the group response times were greater for the cocaine, emotional, or incongruent stimuli $v s$ the corresponding neutral or congruent stimuli. An analysis of word type indicated a significant attentional bias effect for cocaine use words (signed-rank test $S=365.5, p<0.001$ ) and negative emotional words (signed-rank test $S=224.5, p<0.005$ ), as well as a significant cognitive interference effect for incongruent word stimuli $(t=12.3, p<0.001)$. The group relative interference effect was greatest for the word counting Stroop task ( $160 \pm 84 \mathrm{~ms})$, followed by the cocaine Stroop task $(73 \pm 89 \mathrm{~ms})$, and the emotional Stroop task $(25 \pm 55 \mathrm{~ms})$. The individual attentional bias effect for the cocaine and negative emotional word stimuli were highly correlated (Spearman's rho $=0.67, p<0.0001$ ); neither attentional bias effect was correlated with the interference effect for the nondrug-related word counting Stroop task. The plotted reaction time data also illustrate a wide range of interindividual variation in the attentional bias effect for cocaine word stimuli (Figure 1). The attentional bias effect 


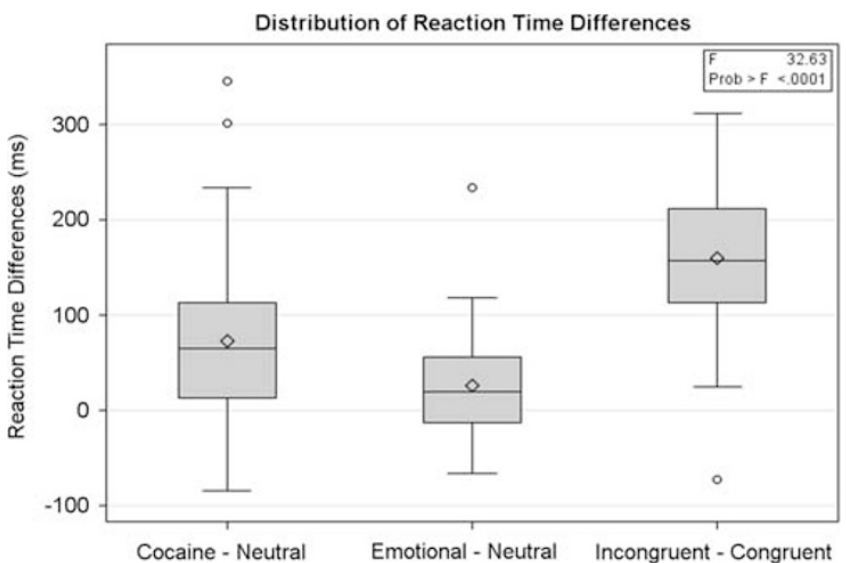

Figure I Attentional and cognitive bias effects for cocaine, negative emotional, and stimulus-incongruent stimuli for cocaine-dependent individuals. Box plots illustrating the mean, median, distribution, and outliers related to the attentional bias effect for cocaine Stroop task (cocStroop, cocaine-neutral), negative emotional Stroop task (eStroop, emotionalneutral), and the related cognitive bias effect for the nondrug-related Stroop task (cStroop, incongruent-congruent) tasks. All Stroop tasks were performed by the same sample of cocaine-dependent men and women and based on a word counting Stroop task designed for $\mathrm{MMRI}$ environments (Bush et al, 1998).

across participants did not correlate significantly with recent cocaine use (past 7 days: $r_{\mathrm{s}}=-0.171, p=0.28$; past 30 days: $r_{\mathrm{s}}=-0.225, p=0.15$ ) or level of addiction-related disability (ASI total score: $r_{s}=0.056, p=0.97$ ).

For the cocaine use-related words there was no significant relationship $(p=0.21)$ between their individual ranking as triggers for drug use and level of attentional bias. Also, level of attentional bias did not differ between the participants who were cocaine positive at fMRI and those who were cocaine negative. Therefore, these two variables were not modeled in the fMRI data analysis of the neural processing correlates of attentional bias for cocaine use stimuli.

\section{fMRI}

Independent component analysis. The recruitment (positive or negative) of independent components to each task condition and the relative increase or decrease in taskrelated activity for each IC for the three condition contrasts of interest are shown in Table 2. Brain regions that were identified as being functionally integrated (both positively and negatively) for each IC and related to cocaine (IC 6, 12, 19), negative emotional (IC 12), or stimulus incongruent (IC 21) conditions are defined in Tables 3 and 4.

Cocaine-neutral word contrast. Of the 30 specified components, 3 were significantly correlated with individual attentional bias for cocaine use stimuli (Figure 2 and Table 3). Attentional bias was positively correlated with activity for two components and negatively with the third. Component 6 (IC 6) was negatively correlated with $\mathrm{AB}-\mathrm{coc}$ $\left(\right.$ rho $=-0.417, p_{\text {uncorr }}=0.0061, p_{\mathrm{FDR}}=0.009$; Figure 2$)$ and consisted of positive contributions from bilateral somatosensory, auditory, and premotor cortices, bilateral insulae, and midline SMA and paracentral lobule as well as negative
Table 3 Locations of Brain Regions Comprising Spatially Independent Networks (IC 6, 12, 19) of Activity Associated with the Contrast of Cocaine-Related and Neutral Cues in CocaineDependent Individuals ${ }^{\mathrm{a}}$

\begin{tabular}{|c|c|c|c|c|c|}
\hline \multirow[t]{2}{*}{$\begin{array}{l}\text { Region } \\
\text { (Brodmann area) }\end{array}$} & \multirow[t]{2}{*}{$\begin{array}{l}\text { Cluster } \\
\text { size } \\
\text { (voxels) }\end{array}$} & \multirow[t]{2}{*}{$\begin{array}{l}\text { Peak voxel } \\
\text { t-score }\end{array}$} & \multicolumn{3}{|c|}{$\begin{array}{c}\text { Peak voxel } \\
\text { MNI } \\
\text { coordinates }\end{array}$} \\
\hline & & & $x$ & $y$ & $\mathbf{z}$ \\
\hline \multicolumn{6}{|l|}{ Component 6} \\
\hline Left postcentral gyrus $(1,2)$ & 1515 & 11.67 & -40 & -20 & 44 \\
\hline Left insula & - & 8.18 & -40 & -18 & 20 \\
\hline Transverse temporal gyrus (4I) & - & 10.62 & -56 & -14 & +12 \\
\hline Right insula & 1159 & 12.58 & 38 & -17 & 18 \\
\hline Right postcentral gyrus (43) & - & 10.19 & 62 & -11 & 15 \\
\hline Right precentral gyrus (6) & - & 11.40 & 53 & -14 & 36 \\
\hline Superior temporal gyrus (22) & - & 6.94 & 65 & -32 & 6 \\
\hline Left inferior frontal gyrus (47) & 50 & -7.80 & -46 & 20 & 0 \\
\hline Right inferior frontal gyrus (47) & 48 & -8.10 & 28 & 22 & -6 \\
\hline Supplementary motor area (6) & 45 & 6.65 & 2 & -11 & 66 \\
\hline Paracentral lobule (5) & 24 & 7.48 & -2 & -38 & 60 \\
\hline \multicolumn{6}{|l|}{ Component 12} \\
\hline Left inferior frontal gyrus (45) & 2455 & 14.48 & -52 & 22 & 12 \\
\hline Dorsomedial prefrontal (9) & - & 12.64 & -4 & 50 & 38 \\
\hline Left middle frontal gyrus (6) & - & $|2.0|$ & -44 & 10 & 50 \\
\hline Left middle temporal gyrus (2I) & 1382 & $|4.3|$ & -64 & -29 & -4 \\
\hline Left superior temporal gyrus (39) & - & 12.48 & -54 & -62 & 26 \\
\hline Right middle frontal gyrus (9) & 408 & -10.33 & 40 & 40 & 30 \\
\hline Left precuneus (7) & 271 & 9.89 & -4 & -52 & 36 \\
\hline Right superior occipital gyrus (19) & 155 & -7.26 & 22 & -74 & 36 \\
\hline Right inferior frontal gyrus (45) & 136 & 9.46 & 50 & 28 & -4 \\
\hline Right middle temporal gyrus (2I) & 133 & 7.48 & 50 & -62 & 24 \\
\hline \multicolumn{6}{|l|}{ Component 19} \\
\hline Left inferior frontal gyrus (45) & $|15|$ & 12.48 & -46 & 16 & 2 \\
\hline Left middle frontal gyrus (9) & - & 7.12 & -32 & 50 & 32 \\
\hline Left insula & - & $|0.8|$ & -40 & -10 & -2 \\
\hline Right inferior frontal gyrus (47) & 1033 & $|1.8|$ & 40 & 20 & -4 \\
\hline Right insula & - & 9.07 & 40 & -12 & -4 \\
\hline Right supramarginal gyrus (40) & 622 & 11.16 & 58 & -44 & 30 \\
\hline Right middle temporal (22) & - & 7.88 & 46 & -46 & 8 \\
\hline Dorsal anterior cingulate cortex (32) & 552 & 9.33 & -2 & 22 & 42 \\
\hline Left superior frontal gyrus (9) & 58 & 7.11 & -32 & 50 & 32 \\
\hline
\end{tabular}

${ }^{a}$ Components thresholded at $|t|>5$ and minimum cluster size of 20 contiguous voxels.

contributions from bilateral inferior frontal cortices (Table 3). Component 19 (IC 19) positively correlated with $\mathrm{AB}-\mathrm{coc} \quad\left(\mathrm{rho}=0.359, \quad p_{\text {uncorr }}=0.0197, \quad p_{\mathrm{FDR}}=0.0197\right.$; Figure 2) and consisted of positive contributions from bilateral inferior frontal cortices (extending to insula), dorsal anterior cingulate (dACC), right supramarginal gyrus extending to middle temporal gyrus, and left dorsolateral 
Table 4 Locations of Brain Regions Comprising a Spatially Independent Network (IC 2I) of Activity Associated with the Contrast of Stimulus-Incongruent and Stimulus-Congruent Trials in Cocaine-Dependent Individuals ${ }^{a}$

\begin{tabular}{|c|c|c|c|c|c|}
\hline \multirow[t]{2}{*}{$\begin{array}{l}\text { Region } \\
\text { (Brodmann area) }\end{array}$} & \multirow{2}{*}{$\begin{array}{c}\text { Cluster } \\
\text { size } \\
\text { (voxels) }\end{array}$} & \multirow{2}{*}{$\begin{array}{c}\text { Peak } \\
\text { voxel } \\
\text { t-score }\end{array}$} & \multicolumn{3}{|c|}{$\begin{array}{l}\text { Peak voxel MNI } \\
\text { coordinates }\end{array}$} \\
\hline & & & $x$ & $y$ & $\mathbf{z}$ \\
\hline Left inferior frontal gyrus (45) & 1564 & 14.00 & -34 & 26 & 8 \\
\hline Left middle frontal gyrus (44) & - & 11.62 & -54 & 16 & 30 \\
\hline Right precuneus (7) & 1121 & 12.11 & 28 & -68 & 36 \\
\hline Right fusiform gyrus (37) & - & 11.23 & 41 & -62 & -13 \\
\hline Left precuneus (7) & 939 & 10.04 & -28 & -70 & 36 \\
\hline Left fusiform gyrus (37) & - & 8.57 & -42 & -62 & -16 \\
\hline Right middle frontal gyrus (9) & 839 & 12.54 & 46 & 10 & 38 \\
\hline Superior frontal gyrus (8) & 555 & 17.20 & -2 & 17 & 54 \\
\hline Right anterior insula & 83 & 11.21 & 34 & 22 & 6 \\
\hline Right lingual gyrus (19) & 43 & 6.65 & 14 & -46 & 0 \\
\hline Dorsal anterior cingulate cortex (24) & 32 & 8.99 & 2 & 8 & 33 \\
\hline Left lingual gyrus (19) & 27 & 6.46 & -23 & -44 & -7 \\
\hline Posterior cingulate (23) & 27 & -6.36 & 4 & -50 & 30 \\
\hline
\end{tabular}

${ }^{a}$ Components thresholded at $|t|>5$ and minimum cluster size of 20 contiguous voxels.

prefrontal cortex (dlPFC; Table 3). Component 12 (IC 12) was also positively correlated ( $\mathrm{rho}=0.448, p_{\text {uncorr }}=0.0029$, $p_{F D R}=0.0087$; Figure 2) and consisted of positive contributions from dorsomedial prefrontal cortex (dmPFC), left inferior and middle frontal cortices, left middle and superior temporal gyri, right middle temporal gyrus, and left precuneus, as well as negative contributions from right middle frontal gyrus and right superior occipital gyrus (Table 3). IC 6, IC 12, and IC 19 accounted for $17 \%, 20 \%$, and $13 \%$ respectively, of the uncontrolled variance in attentional bias effect for the sample. Using multiple regression and controlling for variables of no interest, IC 6, IC 12, and IC 19 accounted for $32 \%, 22 \%$, and $15 \%$ respectively of the variance in attentional bias effect for the sample (Table 5).

Emotional-neutral word contrast. Only 1 of the 30 specified components was significantly correlated with individual attentional bias for negative emotional stimuli. Similar to its relationship to attentional bias for cocaine stimuli, activity for component 12 (IC 12) was also positively correlated (rho $=0.381, p=0.0128$; Figure 3 ) with attentional bias for negative emotional stimuli. IC 12 accounted for $9 \%$ of the uncontrolled variance in the attentional bias effect for negative emotional word stimuli. However, this relationship became marginally nonsignificant $(\beta=7.87$, $p=0.053)$ when controlling for variables of no interest.

Incongruent-congruent counting word contrast. Of the 30 specified, only 1 component was significantly correlated with the individual Stroop effect for the word counting Stroop task (Table 4). Component 21 (IC 21) was positively correlated (rho $=0.399, p=0.0088$; Figure 4 ) and consisted of positive contributions from bilateral dlPFC, dmPFC, bilateral precuneus/occipital cortex extending into fusiform gyri, bilateral lingual gyri, left inferior frontal cortex (IFC), and dACC, as well as negative contributions from posterior cingulate. This component accounted for $16 \%$ of the uncontrolled variance in the Stroop interference effect and $23 \%$ of the variance when controlling for variables of no interest (Table 5). Activity for IC 21 did not correlate with the attentional bias effect for cocaine word stimuli $(r=-0.254, p=0.10)$.

\section{Main effect of task: brain-wide activation and behavioral correlation}

Cocaine-neutral word contrast. Compared with drug use-neutral word stimuli, the personalized cocaine use words were associated with significant activation $(p<0.05$, corrected) of the dlPFC, dmPFC, and ventromedial (vmPFC) prefrontal cortex, posterior cingulate cortex (PCC), right IFC, and right occipitotemporal cortex (Table 6 and Supplementary Figure 1). The results of brain-wide behavioral correlation analyses indicated that the activity of distributed brain regions were significantly ( $p<0.05$, corrected) correlated with the individual level of attentional bias effect for cocaine use-related word stimuli. Positively correlated regions included the occipitotemporal, frontal, and parietal cortex, cerebellum, ventral anterior cingulate cortex, and bilateral premotor cortex, whereas activity within the superior temporal sulcus and motor cortex were negatively correlated (Table 7).

Emotional-neutral word contrast. Compared with emotion-neutral word stimuli, negative emotional words were associated with significant activation $(p<0.05$, corrected) of the bilateral fusiform gyrus, hippocampus, and occipital cortex, and significant relative deactivation of the middle temporal and supramarginal gyrus (Table 6 and Supplementary Figure 2). The results of brain-wide behavioral correlation analyses indicated that no brain regions survived the cluster-level correction $(p<0.05$, corrected) threshold for negative emotional word stimuli.

Incongruent-congruent counting word contrast. Compared with congruent word stimuli, task incongruent words were associated with significant activation $(p<0.05$, corrected) of the bilateral precuneus and right middle occipital cortex, and deactivation of the left parahippocampal gyrus and right middle temporal gyrus (Table 6 and Supplementary Figure 3).

\section{DISCUSSION}

The present study sought a characterization of the brainbehavior relationships related to the well-recognized, wide interindividual variation in attentional bias for drug use cues, focusing on a relatively large sample of cocainedependent men and women. Significant correlation was observed between the level of attentional bias and activation for three networks of brain areas identified by the data dimensionality reduction approach of ICA. Complementary information was obtained by voxel-wise analyses that 


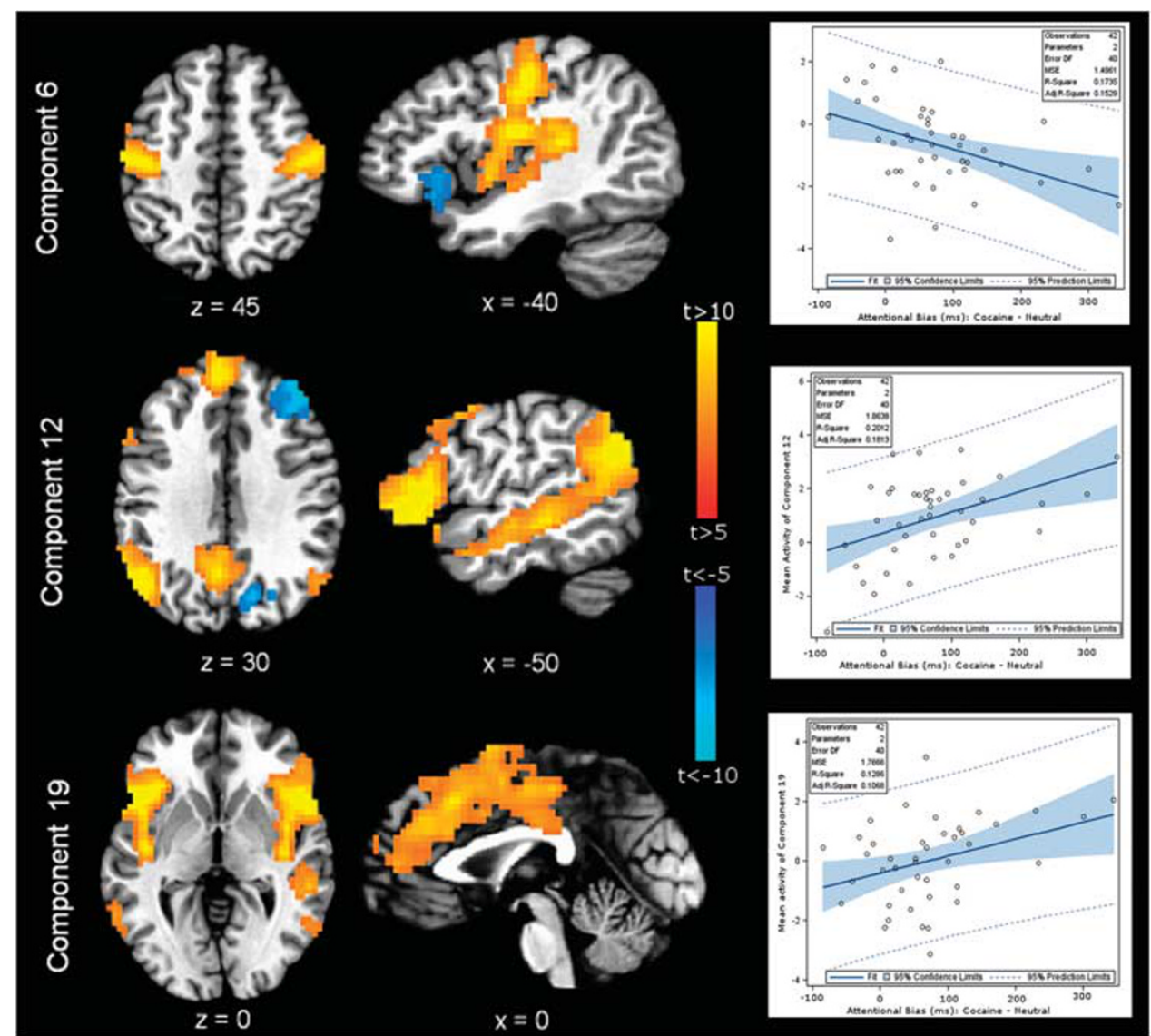

Figure 2 Brain networks whose engagement correlated with individual variation in the attentional bias effect for cocaine use cues. Three of the ICAderived brain networks (IC 6, IC I2, and IC 19) demonstrated task-related activity that significantly correlated with variation in the attentional bias effect for cocaine use cues among cocaine-dependent men and women. Left: statistical maps depict the neural regions comprising each spatially independent network of activated brain areas. Right: regression plots demonstrate significant (all $p<0.05$ ) linear regressions between activity in each network and attentional bias for cocaine stimuli relative to neutral stimuli. A negative relationship (indicating less attentional bias with greater brain activity) was observed for IC 6, and positive relationships were observed for ICs 12 and 19. These relationships were strengthened when controlling for the variables of age, gender, cocaine use in the past 7 days, and impulsiveness.

Table 5 Multivariate Regression Analysis of the Relationship between Component Activity and Attentional (cocStroop) or Cognitive (cStroop) Bias, Controlling for the Effects of Variables of No Interest

\begin{tabular}{|c|c|c|c|c|}
\hline Variable & IC 6 & IC 12 & IC 19 & IC 2 I \\
\hline RT: cocaine-neutral trials & $-4.96(2.04)^{*}$ & $7.84(2.51)$ ***⿻丷木 & $4.99(2.44)^{*}$ & - \\
\hline Age & $0.06(0.02)^{* *}$ & $0.012(0.03)$ & - & - \\
\hline Gender & - & $0.32(0.50)$ & - & - \\
\hline$R^{2}$ & 0.3168 & 0.2146 & 0.1522 & 0.2299 \\
\hline
\end{tabular}

Abbreviations: BIS, Barratt Impulsiveness Scale; Cong, stimulus-congruent trials; IC, independent component; Incong, stimulus-incongruent trials; RT, reaction times. * $p<0.05, * * * 0.01$

identified a group of distributed sites of brain activation as correlated with attentional bias.

The ICA-driven network-level approach identified three networks as related to individual variation in the attentional bias effect. These networks conform in spatial topography to those frequently identified in resting-state and task-related fMRI data sets (Calhoun et al, 2008; Smith et al, 2009; Laird et al, 2013) and suggest that attentional bias involves their 
attributed processes of perception and higher cognitive functioning. The activity of one component (IC 6) exhibited a significant negative relationship with attentional bias, suggesting its role as a regulatory network. The brain areas comprising IC6 were sensory, motor, interoceptive, and social perceptive in nature and accounted for $17 \%$ of the uncontrolled variance in attentional bias effect across the sample. Of these areas, the SMA, as a region involved in controlled action selection (Isoda and Hikosaka, 2007; Rushworth et al, 2002) and response inhibition (Hsu et al, 2011), would be consistent with the engagement of deliberative choice behavior in controlling drug motivation and thus attentional bias. The concordance between IC 6 and an ICA-derived component ('motor network') related to the cognitive control of motor behavior (Zhang and Li, 2012) supports this association. Decreasing attentional bias for drug cues was related to increasing functional connectivity within somatosensory, premotor, auditory, and interoceptive cortex, corresponding to a defined posterior insula network involved in sensorimotor integration (Cauda

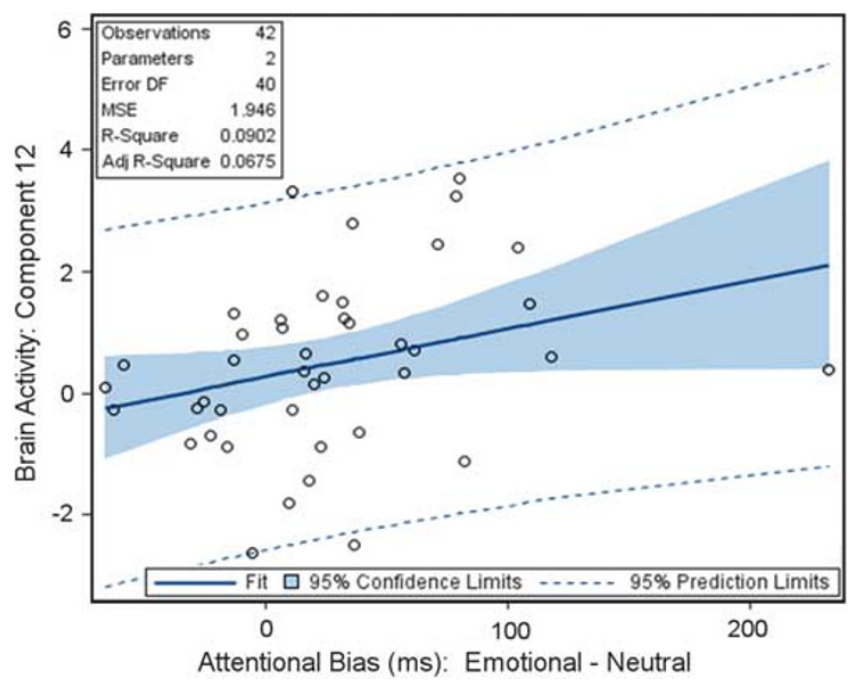

Figure 3 Relationship between network (IC I2) functional connectivity and the attentional bias effect for negative emotional cues. Component 12 brain activity demonstrated a significant $(p=0.012)$ positive relationship with the attentional bias effect for negative emotional stimuli relative to neutral stimuli. However, this relationship became nonsignificant $(p=0.053)$ when controlling for demographic variables. et al, 2011). As an implicit measure of drug motivation, attentional bias for drug cues is thought to capture automatic processes occurring below the level of awareness (Cox et al, 2006; Tiffany, 1990). An interesting, although speculative, inference is that the regulation of drug-related attentional bias involves the elevation of sensory, motor, and interoceptive representations of drug use to conscious awareness, where they can be acted upon. The negative contribution of left and right inferior frontal gyri to this component would thus reflect regions interfering with conscious processing of these drugs cues. This reliance of regulation of drug motivation on attentional awareness is central to recent attempts to use attentional bias modification training (Schoenmakers et al, 2010) and attention control training (Fadardi and Cox, 2009) to reduce drugrelated attentional bias and drug use. Prior studies have demonstrated that drug-dependent individuals can regulate their craving and neural responses by the effortful cognitive regulation of drug cues (Volkow et al, 2010; Kober et al, 2010). This study adds to this literature by demonstrating that engagement of a sensory-motor-dorsal insula network is associated with decreases in the attentional bias effect for implicitly processed cocaine cues.

There is a close association between the processing of drug use-related and negative affective cues in drugdependent individuals (Drobes et al, 2006; Goldstein et al, 2007). This association has been attributed to a major mechanism of relapse in such individuals related to a dysphoric response to drug cue exposure (Koob, 2008; Wheeler et al, 2008). Similar to the findings for smokers (Drobes et al, 2006), there was a strong observed correlation between individual variation in attentional bias effects for drug cue and negative affect stimuli for cocaine-dependent individuals. The present study provides a neural processing network explanation of this association as attentional biases for both cocaine and negative emotional cues were correlated with level of engagement of a frontal-temporalcingulate network (IC 12). Both the middle cingulate and lateral orbital frontal cortex nodes within this network are functionally related to the emotional processing and regulation of aversive states (Singer et al, 2004). The observed increased functional connectivity within this network with increasing attentional bias effect for cocaine cues supports the concept of negative affective responses to drug cue exposure as a precipitant of relapse to drug seeking and use behavior via negative reinforcement

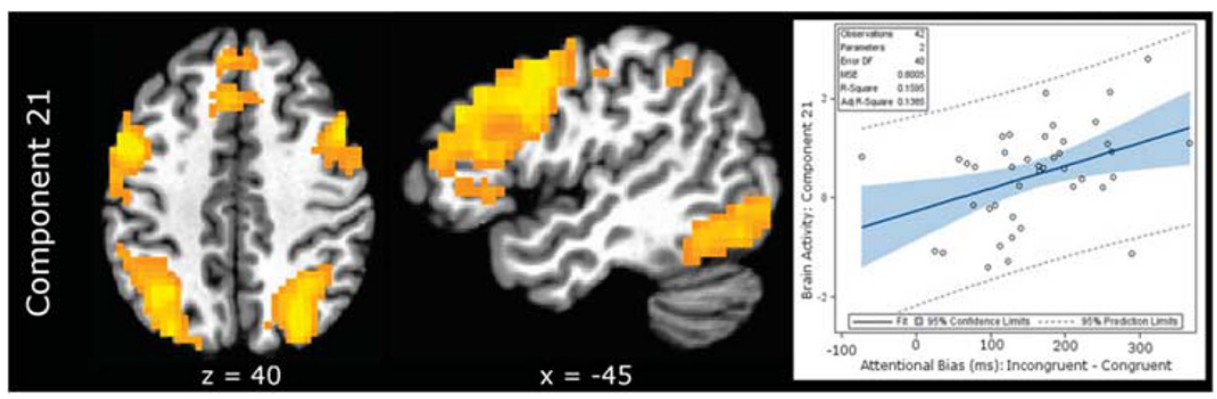

Figure 4 Relationship between network (IC 2I) functional connectivity and the cognitive bias effect for stimulus-incongruent cues. Component 2 I brain activity demonstrated a significant $(p=0.0088)$ positive relationship with the cognitive bias effect for incongruent stimuli relative to congruent stimuli. This relationship was significant $(p<0.0208)$ when controlling for both cocaine use in the last 7 days and impulsiveness. 
Table 6 Locations of Brain Regions of Activity Associated with Univariate Contrast Of Cocaine-Related, Emotion-Related, and Stimulus-Incongruent Stimuli in Cocaine-Dependent Individuals

\begin{tabular}{lccc}
\hline $\begin{array}{l}\text { Region } \\
\text { (Brodmann area) }\end{array}$ & $\begin{array}{c}\text { Cluster size } \\
\text { (no. of } \\
\text { voxels) }\end{array}$ & voxel & $\begin{array}{c}\text { Peak voxel } \\
\text { MNI } \\
\text { coordinates }\end{array}$ \\
\cline { 3 - 5 } & & t-score & $x \quad y \quad z$
\end{tabular}

Cocaine $>$ neutral

Left middle frontal gyrus (8)

Left temporal pole (38)

Left precuneus (19)

Medial superior frontal gyrus (8)

Posterior cingulate cortex (3I)

Right inferior occipital gyrus (18)

Right inferior frontal gyrus (44)

Right inferior frontal gyrus (45)

Right anterior insula

Ventromedial prefrontal cortex (10)

Right fusiform gyrus (37)

Right temporal pole (38)

Posterior cingulate cortex (3I)

Right superior parietal lobule (7)

Right middle temporal gyrus (2I)

Emotion > neutral

Left hippocampus

Left fusiform gyrus (37)

Right middle occipital gyrus (18)

Right fusiform gyrus ( 8 )

Neutral > emotion

Right middle temporal gyrus (2l)

Right supramarginal gyrus (40)

Incongruent > congruent

Right middle occipital gyrus (19)

Left precuneus (19)

Right precuneus (19)

Right middle occipital gyrus (19)

\section{Congruent > incongruent}

Left parahippocampus

Right middle temporal gyrus (2I)
$6.46-44 \quad$ II 45

$5.78-53 \quad 14-10$

$5.64-31-73 \quad 38$

$5.11 \quad-4 \quad 41 \quad 53$

$5.12 \quad-7-55 \quad 23$

$5.16 \quad 32-88-4$

$\begin{array}{llll}5.88 & 50 & 14 & 12\end{array}$

$\begin{array}{llll}5.71 & 53 & 29 & 20\end{array}$

$\begin{array}{llll}5.03 & 26 & 26 & -7\end{array}$

$\begin{array}{llll}5.43 & -7 & 62 & 8\end{array}$

$4.50 \quad 35-37-19$

$4.48 \quad 50 \quad 14-28$

$5.83-10-43 \quad 35$

$3.86 \quad 26-61 \quad 47$

$4.09 \quad 53-25-7$

$4.58-34-38-5$

$4.90-25-79-16$

$\begin{array}{lll}4.67 & 23-85 & 17\end{array}$

$4.44 \quad 14-85-13$

$\begin{array}{llll}81 & 4.40 & 62-49 & 5 \\ 20 & -3.93 & 56-46 & 32\end{array}$

$20 \quad-3.93 \quad 56-46 \quad 32$

$\begin{array}{rrrr}126 & 5.38 & 32-82 & -7 \\ 53 & 4.13 & -10-70 & 44 \\ 20 & 4.51 & 20-73 & 41 \\ 19 & 3.97 & -37-85 & 5\end{array}$

mechanisms (Wheeler et al, 2008; Koob, 2009). Whether this association is because of a direct negative affective response to drug cues or reflects a response to conflict between drug craving and drug unavailability in the MRI setting is not known.

Greater activity within an inferior frontal-parietal-ventral insula network (IC 19) also predicted greater attentional bias effect for cocaine cues. Many regions of IC 19
Table 7 Locations of Brain Regions of Activity Significantly $\left(P_{F D R}<0.05\right)$ Correlated with Attentional Bias Effects for CocaineRelated Stimuli in Cocaine-Dependent Individuals

\begin{tabular}{|c|c|c|c|c|c|}
\hline \multirow[t]{2}{*}{$\begin{array}{l}\text { Region } \\
\text { (Brodmann area) }\end{array}$} & \multirow{2}{*}{$\begin{array}{c}\text { Cluster size } \\
\text { (no. of } \\
\text { voxels) }\end{array}$} & \multirow{2}{*}{$\begin{array}{c}\text { Peak } \\
\text { voxel } \\
\text { t-score }\end{array}$} & \multicolumn{3}{|c|}{$\begin{array}{c}\text { Peak voxel } \\
\text { MNI } \\
\text { coordinates }\end{array}$} \\
\hline & & & $x$ & $y$ & $\mathbf{z}$ \\
\hline \multicolumn{6}{|l|}{ Cocaine $>$ neutral } \\
\hline Right calcarine gyrus (I7) & 311 & 5.56 & || & -85 & 8 \\
\hline Ventromedial prefrontal cortex (I0) & 247 & 5.20 & -1 & 53 & 4 \\
\hline Left inferior frontal gyrus (44) & 117 & 4.96 & -46 & 23 & 8 \\
\hline Right inferior angular gyrus (39) & 115 & 4.92 & 47 & -49 & 15 \\
\hline Pre-SMA (8) & 113 & 4.63 & -4 & 14 & 65 \\
\hline Left cerebellum & 105 & 5.08 & -31 & -67 & -19 \\
\hline Right fusiform gyrus & 80 & 4.99 & 44 & -61 & -10 \\
\hline Left inferior parietal cortex (7) & 59 & 5.10 & -55 & -43 & 38 \\
\hline Right lateral motor cortex (6) & 57 & 5.39 & 35 & -1 & 50 \\
\hline Left middle frontal gyrus (45) & 53 & 4.54 & -52 & 23 & 29 \\
\hline Left middle temporal gyrus (22) & 51 & 4.76 & -52 & -43 & 2 \\
\hline Right temporal pole (38) & 31 & 4.20 & 35 & 14 & -25 \\
\hline Left superior parietal lobule (7) & 30 & 4.94 & -22 & -67 & 38 \\
\hline Right inferior parietal cortex (7) & 28 & 4.96 & 56 & -40 & 38 \\
\hline Left temporal pole (38) & 22 & 4.15 & -34 & 17 & -22 \\
\hline Subgenual cingulate (25) & 21 & 4.88 & 5 & 23 & -7 \\
\hline \multicolumn{6}{|l|}{ Neutral > cocaine } \\
\hline Left superior temporal sulcus (43) & 38 & 4.59 & -53 & -7 & 5 \\
\hline Right superior temporal sulcus (22) & 25 & 5.04 & 59 & -34 & 14 \\
\hline Left primary motor cortex (4) & 23 & 4.41 & 56 & -10 & -1 \\
\hline
\end{tabular}

(including left inferior frontal and right supramarginal gyrus) were also captured by the whole-brain univariate regression analysis, suggesting that their positive association with the cocaine attentional bias effect reflects their integrated activation. As to the functional attributes of the activation of IC 19 with increasing drug cue attentional bias, this network of brain areas comprises a ventral attention network involving the temporoparietal junction (TPJ), insula, and inferior frontal gyrus (Corbetta et al, 2008; Kucyi et al, 2012). This network is involved in the orienting of stimulus-driven attention (Chica et al, 2011) and the detection of salience for behaviorally relevant stimuli (Downar et al, 2002). The direct relationship of activity for this network and increasing cocaine attentional bias suggests that the varying attribution of salience and attention to cocaine stimuli resulted in individual variation in the cocaine attentional bias effect. IC 19 also involved left dorsolateral prefrontal-anterior cingulate cortex connectivity that represented activation of a dorsal attention network (Corbetta et al, 2008). A lesser activation of these regions relative to those comprising a ventral attention network is consistent with their interaction in attentional control and the dampening effect of covert attention on the externally directed task-related attention (Weissman and Prado, 2012). The observed increasing attentional bias effect for cocaine 
cues with increasing activation of IC 19 is consistent with the engagement of covert attention to drug-related stimuli as an 'interrupt signal' that interferes with ongoing taskrelated attention and performance (Corbetta et al, 2008).

The cognitive bias and neural responses to the classic drug-neutral Stroop tasks predict treatment outcomes for cocaine-dependent individuals (Brewer et al, 2008; Streeter et al, 2008). However, individual variation in the cocaine attentional bias effect was unrelated to cStroop task performance or to activity for the cStroop-related neural processing network (IC 21). Individual differences in cognitive control defined by the cStroop task also did not correlate with activity for networks related to the cocaine or negative emotional Stroop tasks. These results support the independence of attentional bias for drug stimuli from general cognitive control ability among cocaine-dependent adults. For the contrast of incongruent and congruent stimulus trials for the word counting Stroop task, an ICAdriven network-level analysis revealed a single network (IC 21) involving the middle, medial and inferior frontal cortices, precuneus, insula, and inferior parietal cortex whose activity was associated with greater interference effects for stimulus incongruent trials. The brain areas comprising this network resemble closely those identified by previous whole-brain mass univariate analyses of the Stroop interference effect in normative subject samples (Bush et al, 1998; Brewer et al, 2008; Polk et al, 2008). However, the voxel-level characterization of bilateral occipital and precuneus activations for cStroop task performance in this sample of cocaine-dependent individuals differed from those previously reported for normative samples. Group comparisons were not the focus of this study, although this deviation is consistent with an effect of cocaine dependence on the neural network response to cognitive control demands posed by Stroop tasks (BarrosLoscertales et al, 2011; Worhunsky et al, 2013).

The observed interindividual variation in the attentional bias effect for personalized drug cues is consistent with that observed for other attentional bias paradigms and drugdependent populations (Janes et al, 2010). Such variance is consistent with wide individual variation in the motivational properties of cocaine; perhaps attributable to variation in the propensity to attribute incentive salience to reward cues in general (Saunders and Robinson, 2011). This variance probably contributes to the lack of significant (corrected) activations for drug $v s$ neutral stimuli often noted for univariate brain-wide or region-of-interest analyses (Goldstein et al, 2007; Kang et al, 2012; Marhe et al, 2013). However, the present study was associated with an unexpectedly robust and distributed pattern of neuroactivation for the brain-wide contrast of cocaine and neutral words. The whole-brain analyses identified frontal, cingulate, parietal, and temporal cortical activations that mirrored a subset of the brain areas involved in the patterns of activation identified by the ICA. The observed mass univariate whole-brain activations related to cocaine use words share dorsal, medial and inferior frontal, and temporal, parietal, and posterior cingulate cortical activations with that of a previous fMRI study of attentional bias for drug-related words in stimulant-dependent individuals (Ersche et al, 2010). The posterior cingulate, medial and inferior frontal cortex, and cerebellum activations are shared by cigarette smokers exposed to smoking cues (Wilson et al, 2012). These collective findings suggest that the brain response to drug use reminders among drugdependent individuals is associated with common attentional, motivational, and motor responses.

If drug-related attentional bias represents a valid cognitive marker of relapse risk among a drug-dependent population, the present study suggests novel neurobiological models of interindividual variation in $\mathrm{AB}-\mathrm{coc}$ and thus risk for relapse. Therefore, the clinical relevance of the study findings is dependent on the clinical relevance of AB$\operatorname{coc}$ and is thus indirect. The study supports a hypothesis that individual variation in the attentional bias effect for cocaine stimuli was predicted by the functional activity of multiple neural processing networks. However, the study has clear limitations that render its greater value to that of hypothesis generation rather than testing. Although a relatively large sample size of 42 subjects was studied, research into individual differences typically demands larger sample sizes to draw meaningful conclusions. Moreover, head motion resulted in the loss of nine possible participants from data analysis and thus diminished the experimental power of the study. The need to replicate the observed brain-behavior relationships is of high priority. As a study of individual differences, the study was based exclusively on the study of a sample of cocaine-dependent individuals. A comparison sample of nondrug-abusing individuals would have provided further useful insights into the comparative organization of normative neural responses to cognitive control demands for negative emotional and nondrugrelated cognitive control processing. Moreover, although cocaine use-related and negative affective stimuli were compared, other behavioral reinforcers (eg, food, sex) and drive states (eg, hunger, arousal) were not considered in a broader characterization of the impact of cocaine addiction on behavioral reinforcement mechanisms. The studied subject sample represented a mixed-sex sample (predominantly male) and therefore was not powered for a characterization of possible sex-dependent differences in the neural correlates of drug cue attentional bias associated with cocaine dependence. Although the study participants were treatment motivated by self-report, less than twothirds subsequently engaged in treatment. Therefore, the clinical significance of the study is uncertain as the neural processing representations of attentional bias were not directly assessed for their possible relatedness to treatment outcomes, abstinence motivation, or drug availability.

\section{CONCLUSIONS}

The ICA-driven fMRI data dimensionality reduction approach allowed the identification of multiple neural processing networks that underlie the putative individual differences in attentional bias for drug use stimuli in cocaine-dependent individuals. Distinct, perhaps opposing, networks mediated individual variation by processing the attentional, salience, and aversive states of cocaine cues vs the engagement of cognitive control and sensory representations of drug use. Given the relationship between measures of attentional bias for drug cues and risk for relapse, the present results suggest that variation in the 
relative engagement of the differing neural processing networks mediates risk for relapse among cocaine-dependent individuals. Future research may enhance the currently poor predictive power of biomarkers of relapse by testing the relatedness of network interactions to treatment outcomes and the longitudinal course of drug abuse.

\section{FUNDING AND DISCLOSURE}

Support for this study was provided by 1T32 DA 022981, 5 R01 DA019999, 5 R21 DA025243, and UL1 TR000039. CDK served as a member of a scientific advisory meeting for Allergan Pharmaceuticals and as a member of the national advisory board for Skyland Trail. He is also a co-holder of US Patent No. 6,373, 990 (Method and device for the transdermal delivery of lithium). CDK received research funding from R01 DA01999, R21 NS065937, 1R21 MH097784, and 2 U19 MH069056-06A1. GAJ received research funding from KL2 TR000063 and UL1 TR000039. JMC received research funding from 1R21 MH097784, 1T32 DA 022981, and UL1 TR000039. ALE received research funding from 1T32 DA 022981. AK received funding from 1F31 DA025491. The authors declare no conflict of interest.

\section{ACKNOWLEDGEMENTS}

We thank our anonymous reviewers for their constructive feedback.

\section{REFERENCES}

Anderson BA, Laurent PA, Yantis S (2011). Value-driven attentional capture. Proc Nat Acad Sci USA 108: 10367-10371.

Ashburner J, Friston KJ (1999). Nonlinear spatial normalization using basis functions. Hum Brain Mapp 7: 254-266.

Ataya AF, Adams S, Mullings E, Cooper RM, Attwood AS, Munafò MR (2012). Internal reliability of measures of substance-related cognitive bias. Drug Alcohol Depend 121: 148-151.

Barros-Loscertales A, Bustamante JC, Ventura-Campos N, Llopis JJ, Parcet MA, Avila C (2011). Lower activation in the right frontoparietal network during a counting Stroop task in a cocaine-dependent group. Psychiatry Res 194: 111-118.

Beckmann CF, Smith SM (2004). Probabilistic independent component analysis for functional magnetic resonance imaging. IEEE Trans Med Imaging 23: 137-152.

Bradley BP, Mogg K, Wright T, Field M (2003). Attentional bias in drug dependence: vigilance for cigarette-related cues in smokers. Psychol Addict Behav 17: 66-72.

Brewer JA, Worhunsky PD, Carroll KM, Rounsaville BJ, Potenza MN (2008). Pretreatment brain activation during stroop task is associated with outcomes in cocaine-dependent patients. Biol Psychiatry 64: 998-1004.

Bush G, Whalen PJ, Rosen BR, Jenike MA, McInerney SC, Rauch SL (1998). The counting Stroop: an interference task specialized for functional neuroimaging-validation study with functional MRI. Hum Brain Mapp 6: 270-282.

Calhoun VD, Adali T, McGinty VB, Pekar JJ, Watson TD, Pearlson GD (2001). fMRI activation in a visual-perception task: network of areas detected using the general linear model and independent component analysis. NeuroImage 14: 1080-1088.

Calhoun VD, Kiehl KA, Pearlson GD (2008). Modulation of temporally coherent brain networks estimated using ICA at rest and during cognitive tasks. Hum Brain Mapp 29: 828-838.
Carpenter KM, Martinez D, Vadhan NP, Barnes-Holmes D, Nunes EV (2012). Measures of attentional bias and relational responding are associated with behavioral treatment outcome for cocaine dependence. Am J Drug Alcohol Abuse 38: 146-154.

Carpenter KM, Schreiber E, Church S, McDowell D (2006). Drug Stroop performance: relationships with primary substance of use and treatment outcome in a drug-dependent outpatient sample. Addict Behav 31: 174-181.

Cauda F, D’Agata FD, Sacco K, Duca S, Gemianini G, Vercelli A (2011). Functional connectivity of the insula in the resting brain. NeuroImage 55: 8-23.

Chica AB, Bartolomeo P, Valero-Cabre A (2011). Dorsal and ventral parietal contributions to spatial orienting in the human brain. J Neurosci 31: 8143-8149.

Congdon E, Mumford JA, Cohen JR, Galvan A, Aron AR, Xue G et al (2010). Engagement of large-scale networks is related to individual differences in inhibitory control. Neuroimage 53: 653-663.

Copersino ML, Serper MR, Vadhan N, Goldberg BR, Richarme D, Chou JCY et al (2004). Cocaine craving and attentional bias in cocaine-dependent schizophrenic patients. Psychiatry Res 128: 209-218.

Corbetta M, Patel G, Shulman GL (2008). The reorienting system of the human brain: from environment to theory of mind. Neuron 58: $306-324$.

Cox RW (1996). AFNI: software for analysis and visualization of functional magnetic resonance neuroimages. Comput Biomed Res 29: 162-173.

Cox WM, Fadardi JS, Pothos EM (2006). The addiction-stroop test: theoretical considerations and procedural recommendations. Psychol Bull 132: 443-476.

Downar J, Crawley AP, Mikulis DJ, Davis KD (2002). A cortical network sensitive to stimulus salience in a neutral behavioral context across multiple sensory modalities. J Neurophysiol 87: 615-620.

Drobes DJ, Elibero A, Evans DE (2006). Attentional bias for smoking and affective stimuli: a Stroop task study. Psychol Addict Behav 20: 490-495.

Ersche KD, Bullmore ET, Craig KJ, Shabbir SS, Abbott S, Muller U et al (2010). Influence of compulsivity of drug abuse on dopaminergic modulation of attentional bias in stimulant dependence. Arch Gen Psychiatry 67: 632-644.

Fadardi JS, Cox WM (2009). Reversing the sequence: reducing alcohol consumption by overcoming alcohol attentional bias. Drug Alcohol Depend 101: 137-145.

First MB, Opler LA, Hamilton RM, Linder J, Linfield LS, Silver JM et al (1993). Evaluation in an inpatient setting of DTREE, a computer-assisted diagnostic assessment procedure. Compr Psychiatry 34: 171-175.

Friston KJ, Ashburner J, Frith CD, Poline JB, Heather JD, Frackowiak RSJ (1995). Spatial registration and normalization of images. Hum Brain Mapp 3: 165-189.

Goldstein RZ, Tomasi D, Rajaram S, Cottone LA, Zhang L, Maloney $\mathrm{T}$ et al (2007). Role of the anterior cingulate and medial orbitofrontal cortex in processing drug cues in cocaine addiction. Neurosci 144: 1153-1159.

Hester R, Dixon V, Garavan H (2006). A consistent attentional bias for drug-related material in active cocaine users across word and picture versions of the emotional Stroop task. Drug Alcohol Depend 81: 251-257.

Hsu TY, Tseng LY, Yu JX, Kuo WJ, Hung DL, Tzeng OJ et al (2011). Modulating inhibitory control with direct current stimulation of the superior medial frontal cortex. Neuroimage 56: 2249-2257.

Isoda M, Hikosaka O (2007). Switching from automatic to controlled action by monkey medial frontal cortex. Nat Neurosci 10: $240-248$.

Janes AC, Pizzagalli DA, Richardt S, Bd Frederick, Holmes AJ, Sousa J et al (2010). Neural substrates of attentional bias for smoking-related cues: an fMRI study. Neuropsychopharmacology 35: 2339-2345. 
Kang OS, Chang DS, Jahng GH, Kim SY, Kim H, Kim JW et al (2012). Individual differences in smoking-related cue reactivity in smokers: an eye-tracking and fMRI study. Prog Neuropsychopharmacol Biol Psychiatry 38: 285-293.

Kilts CD, Schweitzer JB, Quinn CK, Gross RE, Faber TL, Muhammad F et al (2001). Neural activity related to drug craving in cocaine addiction. Arch Gen Psychiatry 58: 334-341.

Kober H, Mende-Siedlecki P, Kross EF, Weber J, Mischel W, Hart CL et al (2010). Prefrontal-striatal pathway underlies cognitive regulation of craving. Proc Natl Acad Sci USA 107: 14811-14816.

Koob GF (2008). A role of brain stress systems in addiction. Neuron 59: 11-34.

Koob GF (2009). Neurobiological substrates for the dark side of compulsivity in addiction. Neuropharmacology 56(Suppl 1): 18-31.

Kucyi A, Hodaie M, Davis KD (2012). Lateralization in intrinsic functional connectivity of the temporoparietal junction with salience- and attention-related brain networks. J Neurophysiol 108: 3382-3392.

Laird AR, Eickhoff SB, Rottschy C, Bzdok D, Ray KL, Fox PT (2013). Networks of task co-activation. NeuroImage 80: 505-514.

Marhe R, Luijten M, van de Wetering BJ, Smits M, Franken IH (2013). Individual differences in anterior cingulate activation associated with attentional bias predict cocaine use after treatment. Neuropsychopharmacology 6: 1085-1093.

Moeller SJ, Maloney T, Parvaz MA, Dunning JP, Alia-Klein N, Woicik PA et al (2009). Enhanced choice for viewing cocaine pictures in cocaine addiction. Biol Psychiatry 66: 169-176.

Paliwal P, Hyman SM, Sinha R (2008). Craving predicts time to cocaine relapse: further validation of the Now and Brief versions of the cocaine craving questionnaire. Drug Alcohol Depend 93: 252-259.

Pantazatos SP, Talati A, Pavlidis P, Hirsch J (2012). Decoding unattended fearful faces with whole-brain correlations: an approach to identify condition-dependent large-scale functional connectivity. PLoS Computat Biol 8: e102441.

Polk TA, Drake RM, Jonides JJ, Smith MR, Smith EE (2008). Attention enhances the neural processing of relevant features and suppresses the processing of irrelevant features in humans: a functional magnetic resonance imaging study of the Stroop task. J Neurosci 28: 13786-13792.

Potenza MN, Hong KI, Lacadie CM, Fulbright RK, Tuit KL, Sinha R (2012). Neural correlates of stress-induced and cue-induced drug craving: influences of sex and cocaine dependence. Am J Psychiatry 169: 406-414.

Rushworth MF, Hadland KA, Paus T, Sipila PK (2002). Role of the human medial frontal cortex in task switching: a combined fMRI and TMS study. J Neurophysiol 87: 2577-2592.

Saunders BT, Robinson TE (2011). Individual variation in the motivational properties of cocaine. Neuropsychopharmacology 36: $1668-1676$.

Scher CD, Stein MB, Asmundson GJG, McCreary DR, Forde DR (2001). The childhood trauma questionnaire in a community sample: psychometric properties and normative data. J Trauma Stress 14: 843-857.

Schoenmakers TM, de Bruin M, Lux IFM, Goertz AG, Van Kerhkof DHAT, Wiers RW (2010). Clinical effectiveness of attentional bias modification training in abstinent alcoholic patients. Drug Alcohol Depend 109: 30-36.

Shadel W, Martino SC, Setodji C, Cervone D, Witkiewitz K, Beckjord EB et al (2011). Lapse-induced surges in craving influence relapse in adult smokers: an experimental investigation. Health Psychol 30: 588-596.

Singer T, Seymour B, O'Doherty J, Kaube H, Dolan RJ, Frith CD (2004). Empathy for pain involves the affective but not sensory components of pain. Science 303: 1157-1162.

Smith SM, Fox PT, Miller KL, Glahn DC, Fox PM, Mackay CE et al (2009). Correspondence of the brain's functional architecture during activation and rest. Proc Natl Acad Sci USA 106: 13040-13405.

Stanford MS, Mathias CW, Dougherty DM, Lake SL, Anderson NE, Patton JH (2009). Fifty years of the Barratt Impulsiveness Scale: an update and review. Personality Individ Diff 47: 383-395.

Streeter CC, Terhune DB, Whitfield TH, Gruber S, Sarid-Segal O, Silveri MM et al (2008). Performance on the Stroop predicts treatment compliance in cocaine-dependent individuals. Neuropsychopharmacology 33: 827-836.

Tiffany ST (1990). A cognitive model of drug urges and drug-use behavior: role of automatic and nonautomatic processes. Psychol Rev 97: 147-168.

Vadhan NP, Carpenter KM, Copersino ML, Hart CL, Foltin RW, Nunes EV (2007). Attentional bias towards cocaine-related stimuli: relationship to treatment-seeking for cocaine dependence. Am J Drug Alcohol Abuse 33: 727-736.

Volkow ND, Fowler JS, Wang G-J, Telang F, Logan J, Jayne M et al (2010). Cognitive control of drug craving inhibits brain reward regions in cocaine abusers. NeuroImage 49: 2536-2543.

Waters A, Marhe R, Franken I (2012). Attentional bias to drug cues is elevated before and during temptations to use heroin and cocaine. Psychopharmacology 219: 909-921.

Weissman DH, Prado J (2012). Heightened activity in a key region of the ventral attention network is linked to reduced activity in a key region of the dorsal attention network during unexpected shifts of covert visual spatial attention. NeuroImage 61: 798-804.

Whalen PJ, Bush G, McNally RJ, Wilhelm S, McInerney SC, Jenike MA et al (1998). The emotional counting Stroop paradigm: a functional magnetic resonance imaging probe of the anterior cingulate affective division. Biol Psychiatry 44: 1219-1228.

Wheeler RA, Twining RC, Jones JL, Slater JM, Grigson PS, Carelli RM (2008). Behavioral and electrophysiological indices of negative affect predict cocaine self-administration. Neuron 57: 774-785.

Wilson SJ, Sayette MA, Fiez JA (2012). Quitting-unmotivated and quitting-motivated cigarette smokers exhibit different patterns of cue-elicited brain activation when anticipating an opportunity to smoke. J Abnorm Psychol 121: 198-211.

Worhunsky PD, Stevens MC, Carroll KM, Rounsaville BJ, Calhoun VD, Pearlson GD et al (2013). Functional brain networks associated with cognitive control, cocaine dependence, and treatment outcome. Psychol Addict Behav 27: 477-488.

Zhang S, Li CS (2012). Functional networks for cognitive control in a stop signal task: independent component analysis. Hum Brain Mapp 33: 89-104.

Supplementary Information accompanies the paper on the Neuropsychopharmacology website (http://www.nature.com/npp) 